\title{
Synthesis of High-Performance Lignin-based
}

\section{Inverse Thermoplastic Vulcanizates with Tailored}

\section{Morphology and Properties}

Nihal Kanbargi ${ }^{a, c *}$, Monojoy Goswami ${ }^{a}$, Liam Collins $^{b}$, Logan T Kearney ${ }^{a}$, Christopher C Bowland ${ }^{a}$, Keonhee Kim ${ }^{c}$, Kalavathy Rajan ${ }^{c}$, Nicole Labbe ${ }^{c *}$, Amit K Naskar ${ }^{a, c *}$

${ }^{a}$ Chemical Sciences Division, Oak Ridge National Laboratory, 1 Bethel Valley Rd, Oak Ridge, TN37831-6053, USA. Email: naskarak@ornl.gov; kanbargin@ornl.gov.

${ }^{\mathrm{b} C e n t e r}$ for Nanophase Materials Sciences, Oak Ridge National Laboratory, 1 Bethel Valley Road, Oak Ridge, TN 37831, USA

${ }^{\mathrm{c} C e n t e r}$ for Renewable Carbon, University of Tennessee, 2506 Jacob Drive, Knoxville, TN 37996, USA. Email: nlabbe@utk.edu; anaskar@utk.edu. 


\section{Supporting Information}

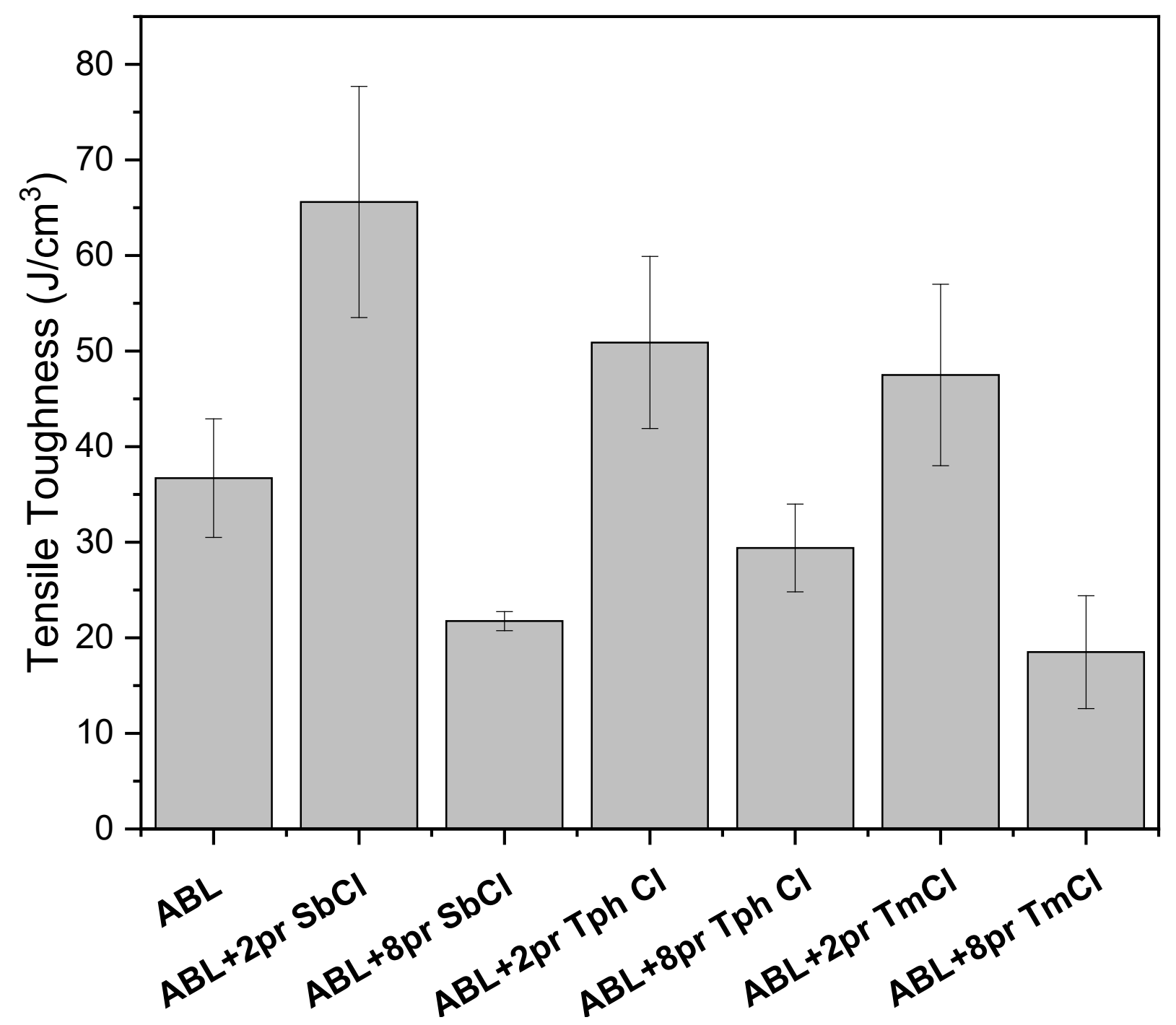

Figure S. 1. Tensile toughness, proportional to the area under the curve plotted as a function of added small molecule

Molecular Dynamics (MD) Simulations: The MD simulations are carried following KremerGrest $^{1}$ bead-spring model of coarse-grained (CG) polymers. This model considers monomers of the polymer as beads connected by finite extendable nonlinear elastic (FENE) spring given by, 
$U^{F E N E}\left(r_{i j}\right)=-1 / 2 K R_{0}^{2} \ln \left(1-r_{i j} / R_{0}\right)^{2}$, where $K$ is the spring constant and $R_{0}$ is the maximum stretching distance between two bonded monomers. Both the chains are modeled as flexible beadspring chains. The coarse-grained NBR chain and a coarse-grained coniferyl alcohol monomeric Lignin unit are shown in Figure S. 1.a. One unit of NBR has two blocks one for Butadiene and another for Acrylonitrile. The cyan color represents the nitrile monomers of NBR. The CG lignin molecules has three phenolic side chains that can crosslink to form larger lignin molecules. We studied three different sets of systems as shown in Figure S. 1.b. The three different small molecules are modeled following experimental structure of the added moieties. System 1 has a flexible chain of 10 monomers as shown in Figure S. 1.b. System 2 consists of a small molecule with three monomers connected at $180^{\circ}$ angles. System 3 consists of 4 small molecules and the central molecule connected to the other 3 molecules at $120^{\circ}$ angle. All the small molecules have harmonic bonds between the monomers. The interactions between all the monomers are governed by Lennard-Jones (LJ) potential given by, $U^{L J}\left(r_{i j}\right)=4 \varepsilon\left[\left(\frac{\sigma}{r_{i j}}\right)^{12}-\left(\frac{\sigma}{r_{i j}}\right)^{6}\right]+\varepsilon$, where $\varepsilon$ is the LJ interaction strength and $\sigma$ is the monomer diameter. The interactions between different types of monomers can be attractive or repulsive. The attractive and repulsive cutoffs are standard LJ values given by $r_{\text {cutoff }}=2.5 \sigma$ for attractive and $1.12 \sigma$ for repulsive respectively. The simulations are carried out using Langevin dynamics, available in LAMMPS MD simulator (lammps.sandia.gov). The equation of motion for Langevin dynamics is shown in following.

$$
\frac{d^{2} \vec{r}}{d t^{2}}=\frac{-\nabla U}{m}-\gamma \frac{d r}{d t}+<R(t) R\left(t^{\prime}\right)>
$$


Where, $\gamma$ is the drag coefficient or friction coefficient and the last term is random stationary force with zero mean. The last term of the above equation is Gaussian White Noise that attaches a stochastic heat bath the system for energy dissipation, thus controlling the temperature of the system. One advantage of Langevin dynamics is the solvent interactions are embedded in the drag
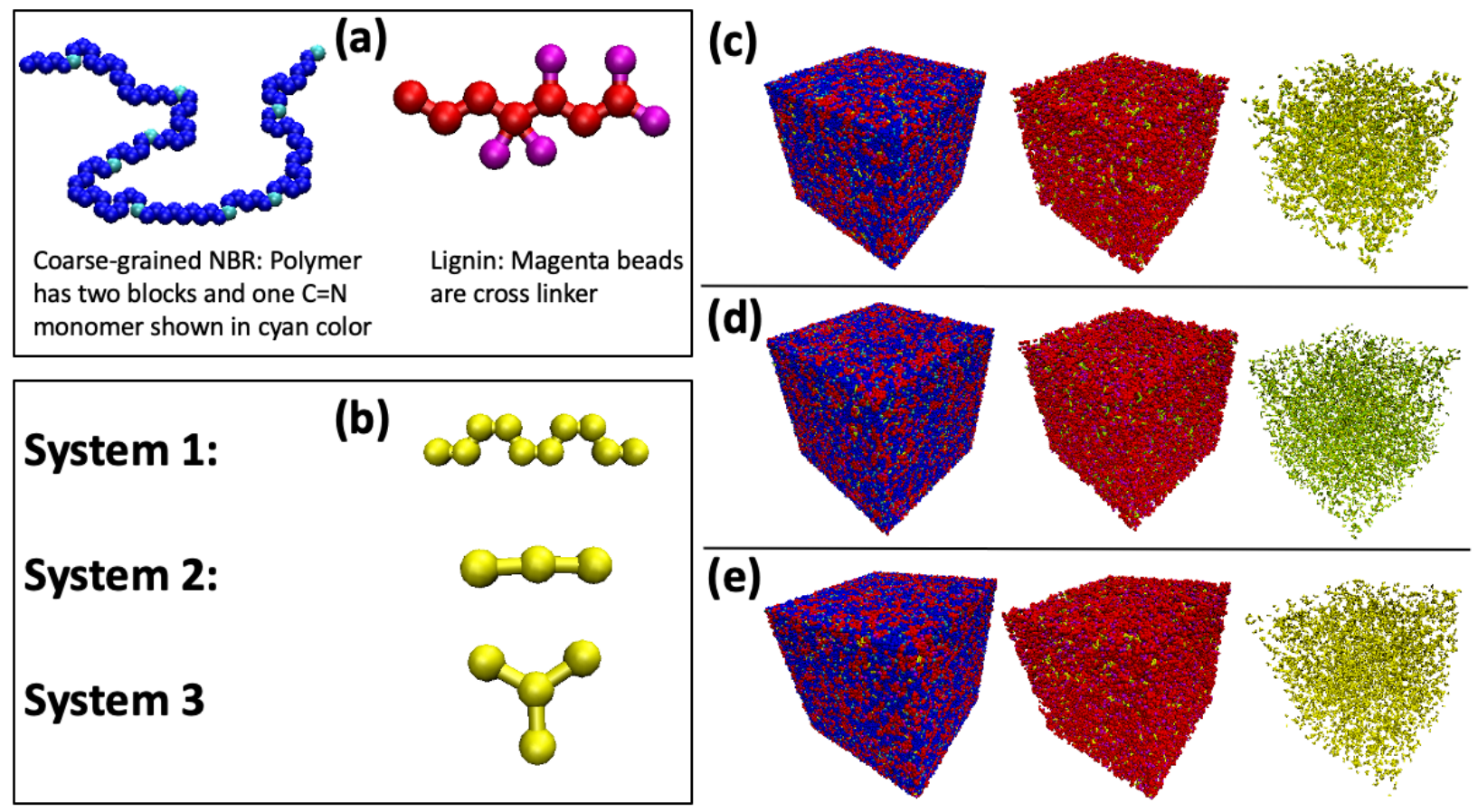

Figure S. 2. Building the model system. (a) The NBR and Lignin molecules in bead-spring polymer model. (b) Kremer-Grest bead-spring model of utilized crosslinkers, (c), (d), (e) initial systems for System 1, 2 and 3 respectively

coefficient, and hence solvent does not need to be explicitly added in cases where solvent is not the focus of the study. This implicit solvent method is computationally efficient, while it can describe the fundamental physics accurately. The initial systems are shown in Figure S. 1 c. The top, middle and the bottom figures are initial systems for System 1, 2 and 3 respectively. The initial systems are randomly generated in a box using Packmol package ${ }^{2}$. The left figures show full system, while the middle and right figures show lignin+small molecule and small molecules respectively, i.e., the NBR CG molecules are deleted to show a clear view of the lignin and lignin and small molecules. The simulations are performed in periodic boundary conditions (PBC) under 
NVT ensemble. All the system parameters, such as time temperature and distance are reported in reduced units. Small molecule distributions are shown for three different systems in Figure S. 2. at the end of simulation, i.e., an equilibrated system.
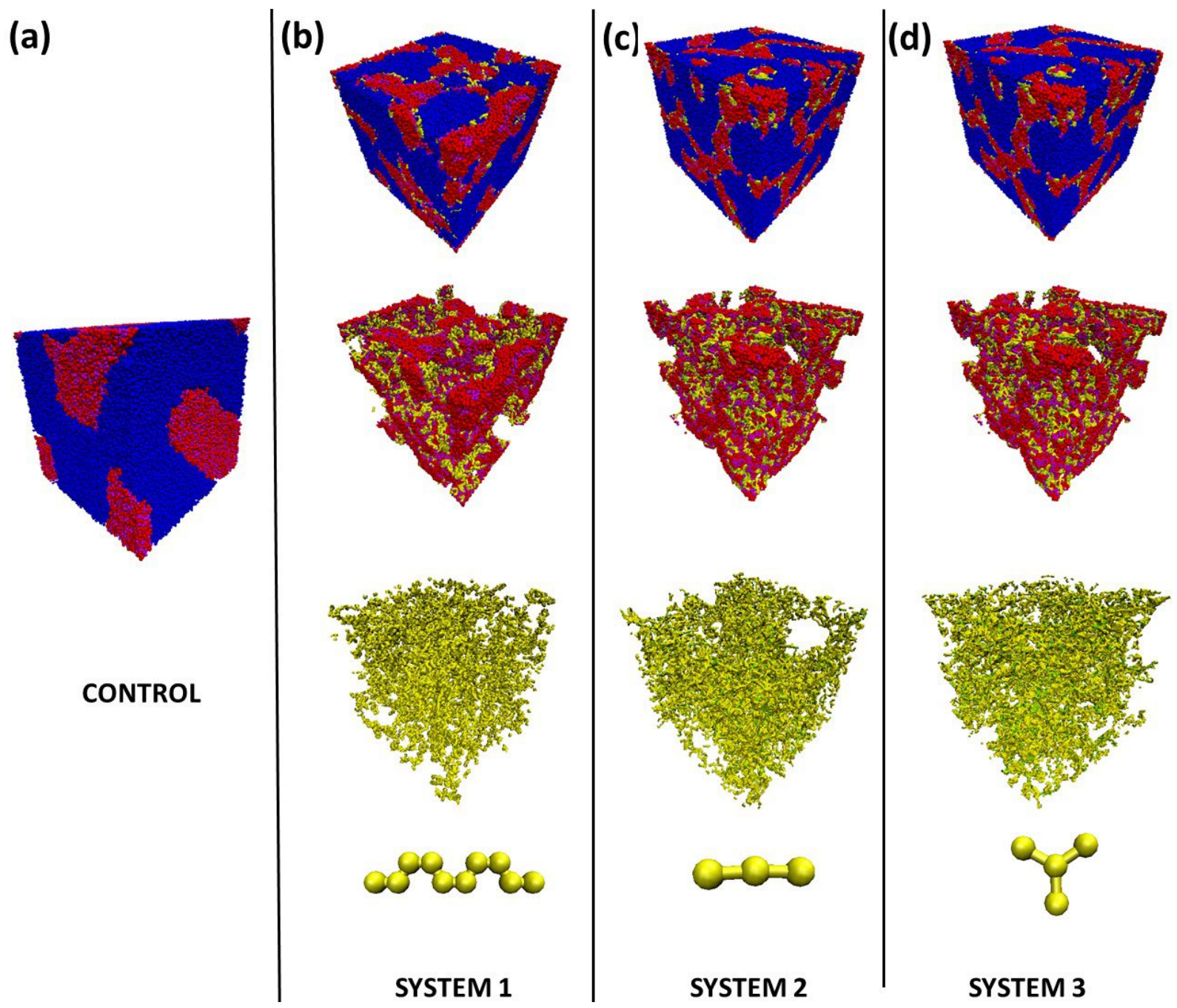

Figure S. 3. 3D images of all the systems. (a) control system. (b), (c) and (d) are drawn with the added small molecules. For the middle panel of (b), (c) and (d) the NBR matrix domains are deleted to show the distribution of lignin and small molecules with the bottom panel showing the distribution of small molecules only. 


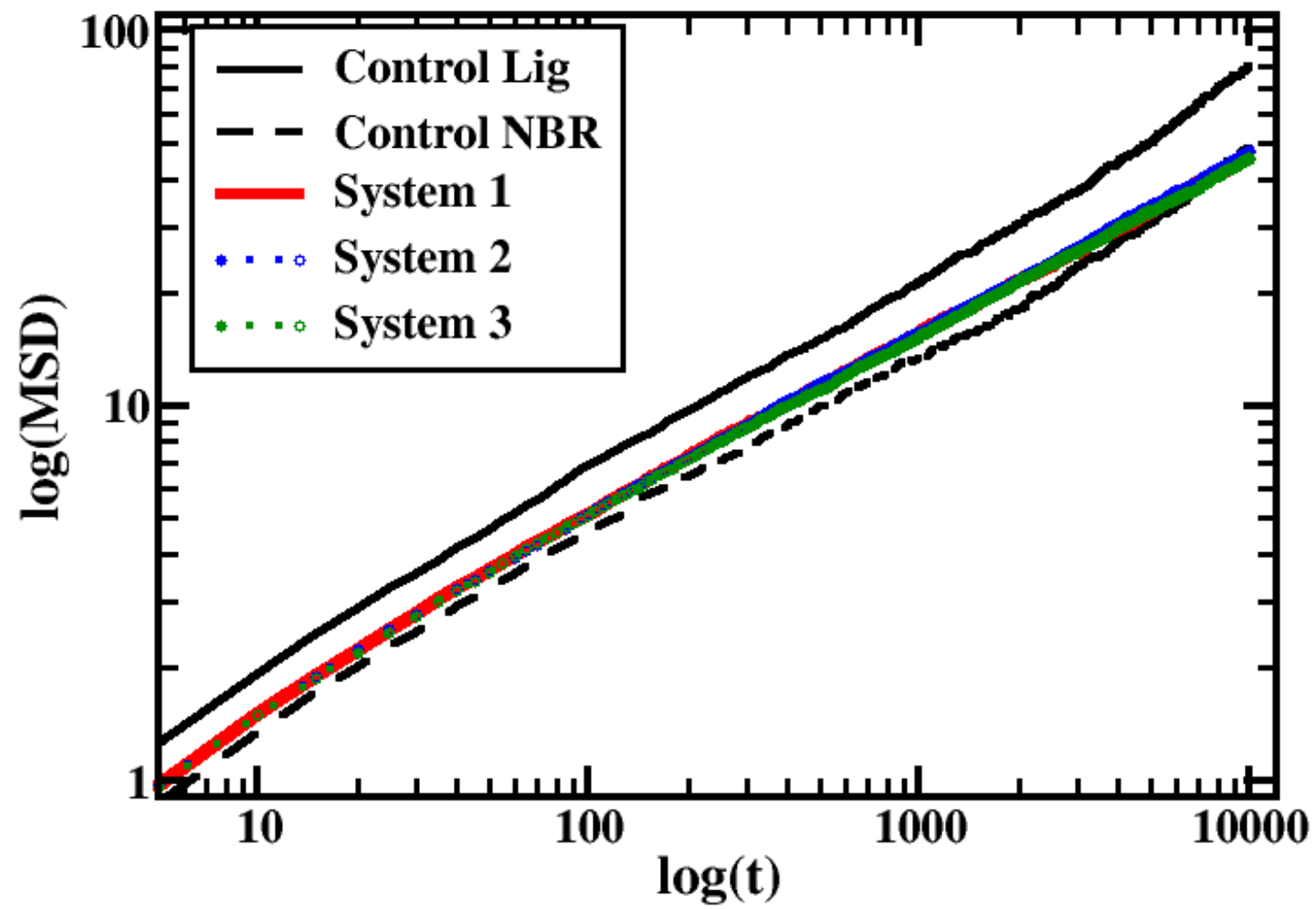

Figure S. 4. MSD plot for NBR in presence of SbCl (black solid line), TphCl (red dotted line) and $\mathrm{TmCl}$ (blue dotted line) respectively, showing very similar, overlapping dynamics with added crosslinkers, since NBR forms the matrix in all the cases and has low interaction with the small molecules. Control NBR (black dashes) and lignin (red solid line) are shown for comparison.

Thermal Analysis, Spectroscopic Characterization, and Microscopy Data: Glass transition temperatures measured by DSC show a similar trend to that of the alpha transitions measured via DMA. The control blend itself shows a single $\mathrm{Tg}$ between that of pure lignin and NBR41, indicating miscibility. Adding crosslinker does seem to shift the Tg to a higher temperature, but the differences here are more difficult to discern. Tgs are also around $15^{\circ} \mathrm{C}$ lower that that measured by DMA, but this is commonly observed. TGA analysis has shown that there is an increase in temperature at which onset of degradation occurs on the samples that are treated with crosslinkers. This is also evident in the derivative of the weight loss vs temperature curve. There appears to be a splitting in the peak observed around $450^{\circ} \mathrm{C}$ in the crosslinked specimens, which 
is not seen in the control $\mathrm{ABL}$, most likely corresponding to the un-crosslinked and crosslinked lignin domains within the blend.
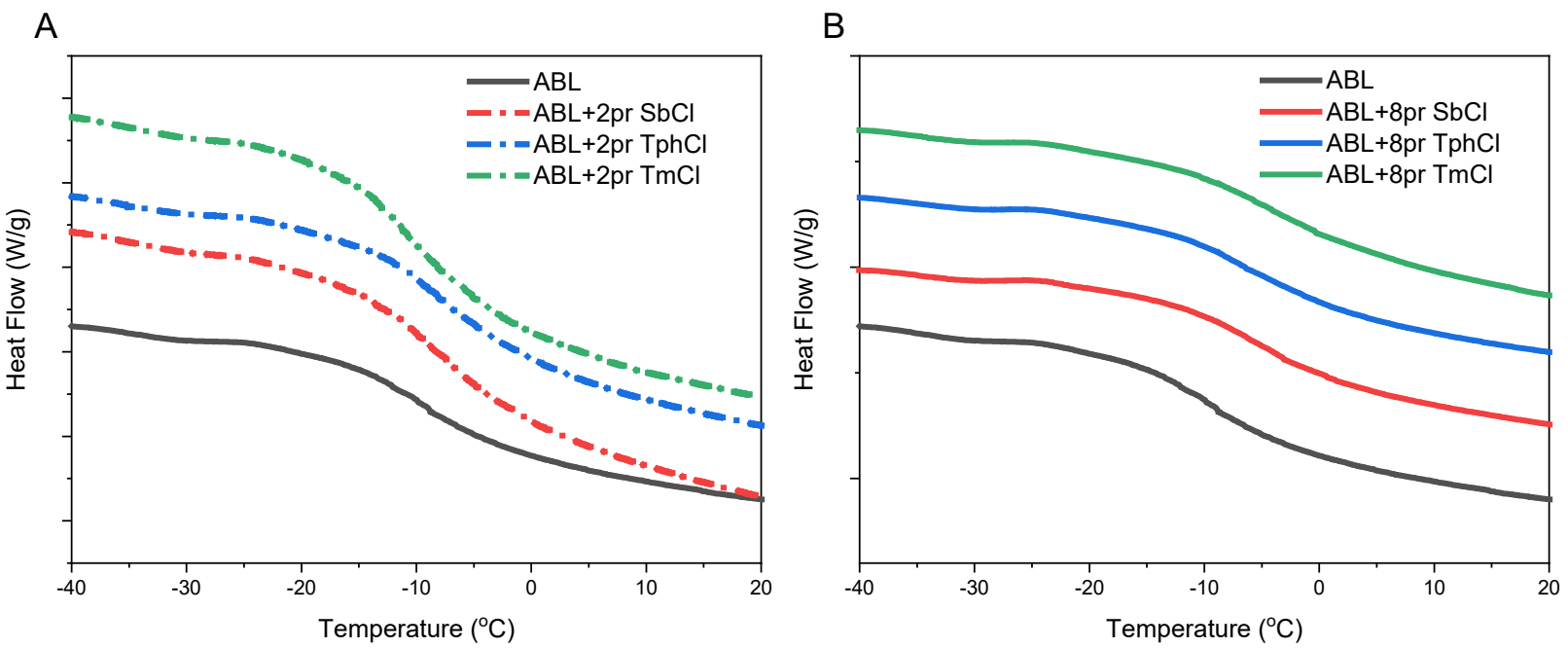

Figure S. 5. DSC showing Tgs of A) ABL with 2pr crosslinkers, B) ABL treated with 8pr crosslinkers
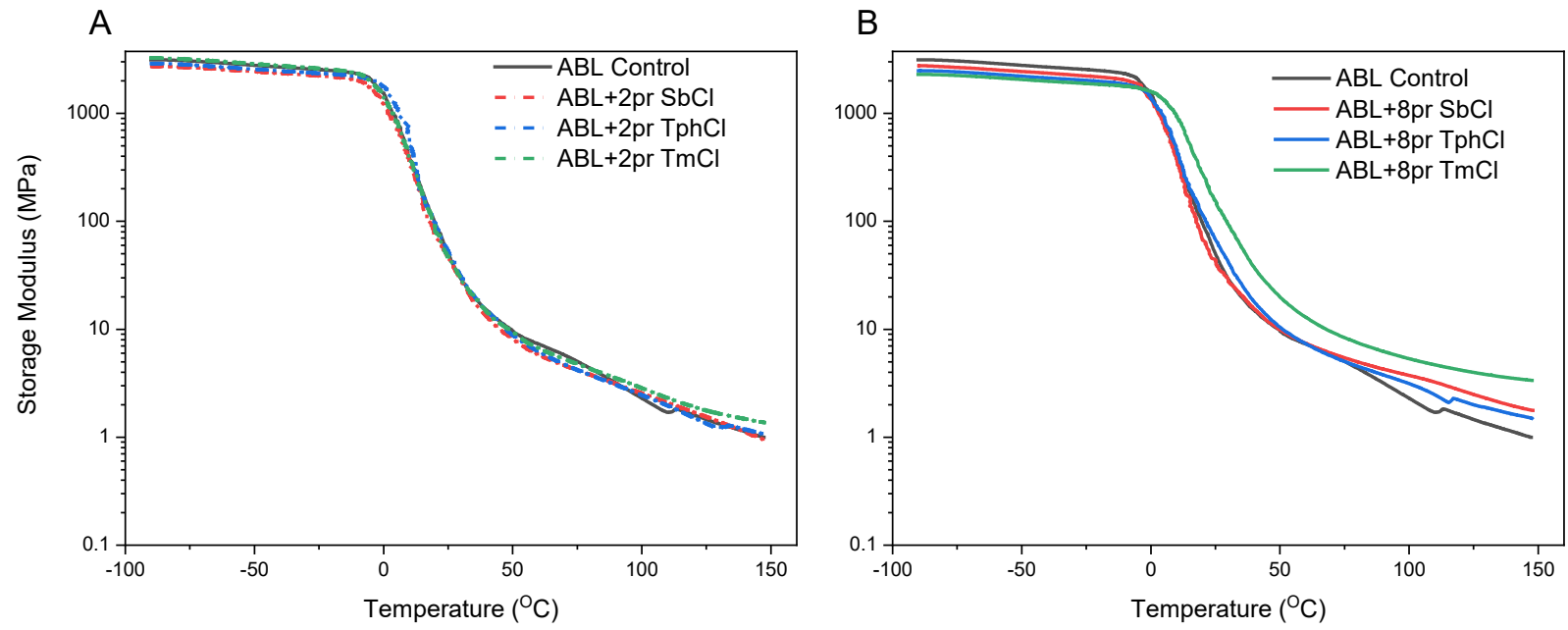

Figure S. 6. Storage modulus of A) ABL treated with 2pr crosslinkers, and B) ABL treated with 8pr crosslinkers 

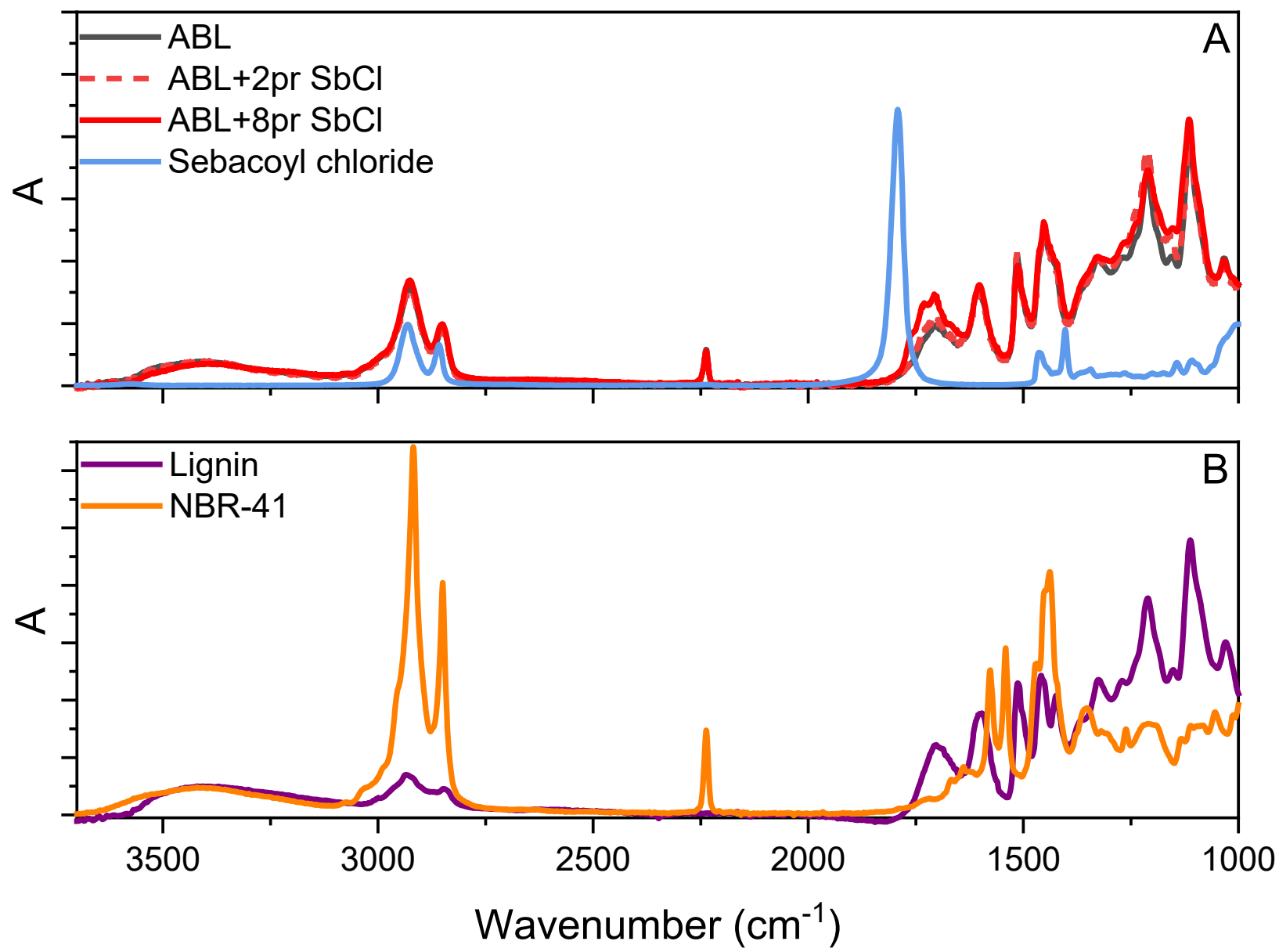

Figure S. 7.A) FTIR Spectra of $\mathrm{SbCl}$ compared with $\mathrm{ABL}+\mathrm{SbCl}$ showing no trace left of sebacoyl chloride indicating compete esterification, B) Spectra of lignin and NBR41 shown for comparison 

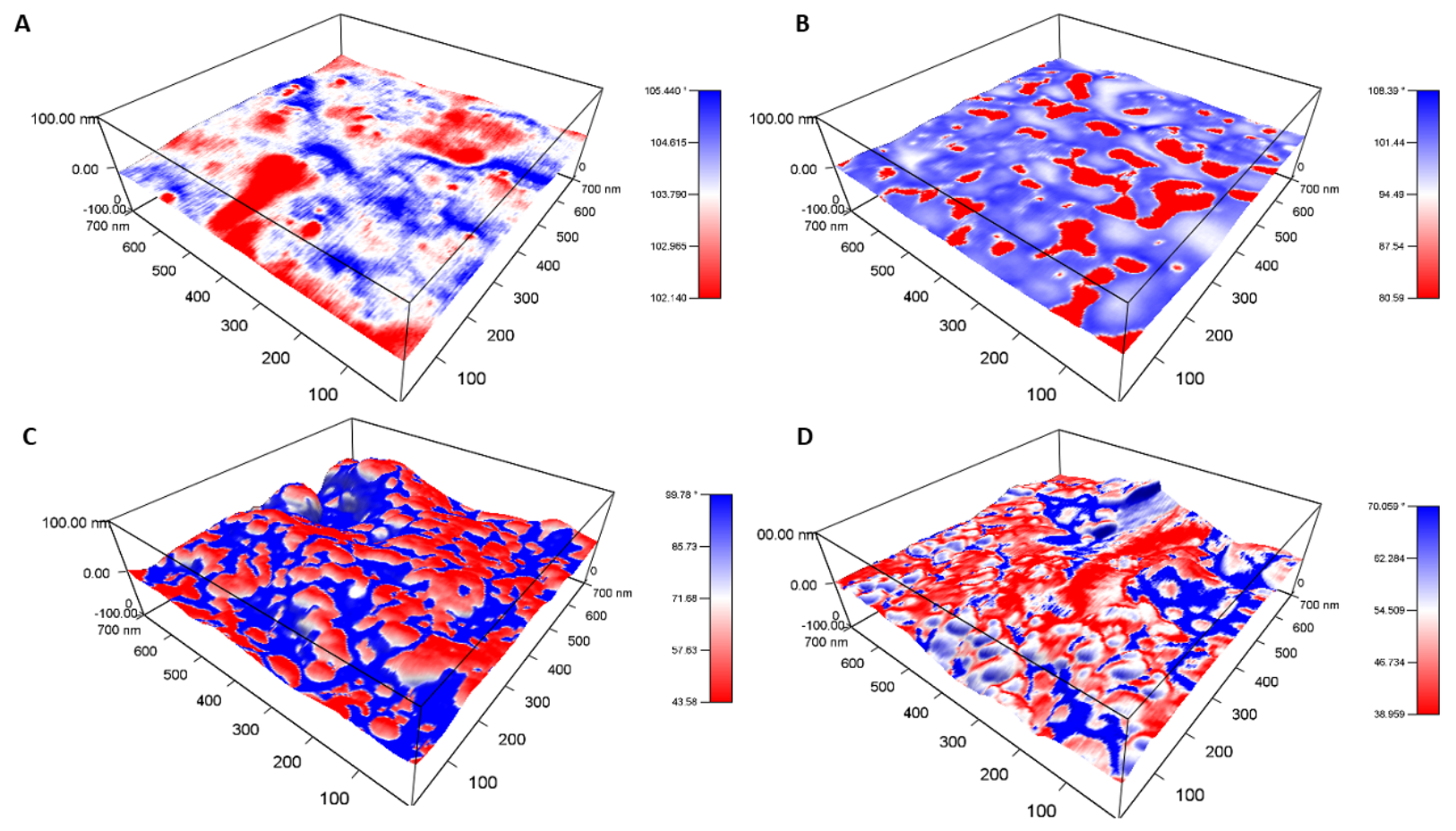

Figure S. 8. Bimodal phase data overlaid over topography for A) control, B) ABL $+2 \mathrm{pr} \mathrm{SbCl,} \mathrm{C)}$ $\mathrm{ABL}+2 \mathrm{pr} \mathrm{TphCl}$ and $\mathrm{D}) \mathrm{ABL}+2 \mathrm{pr} \mathrm{TmCl}$ indicating increased roughness with crosslinker 

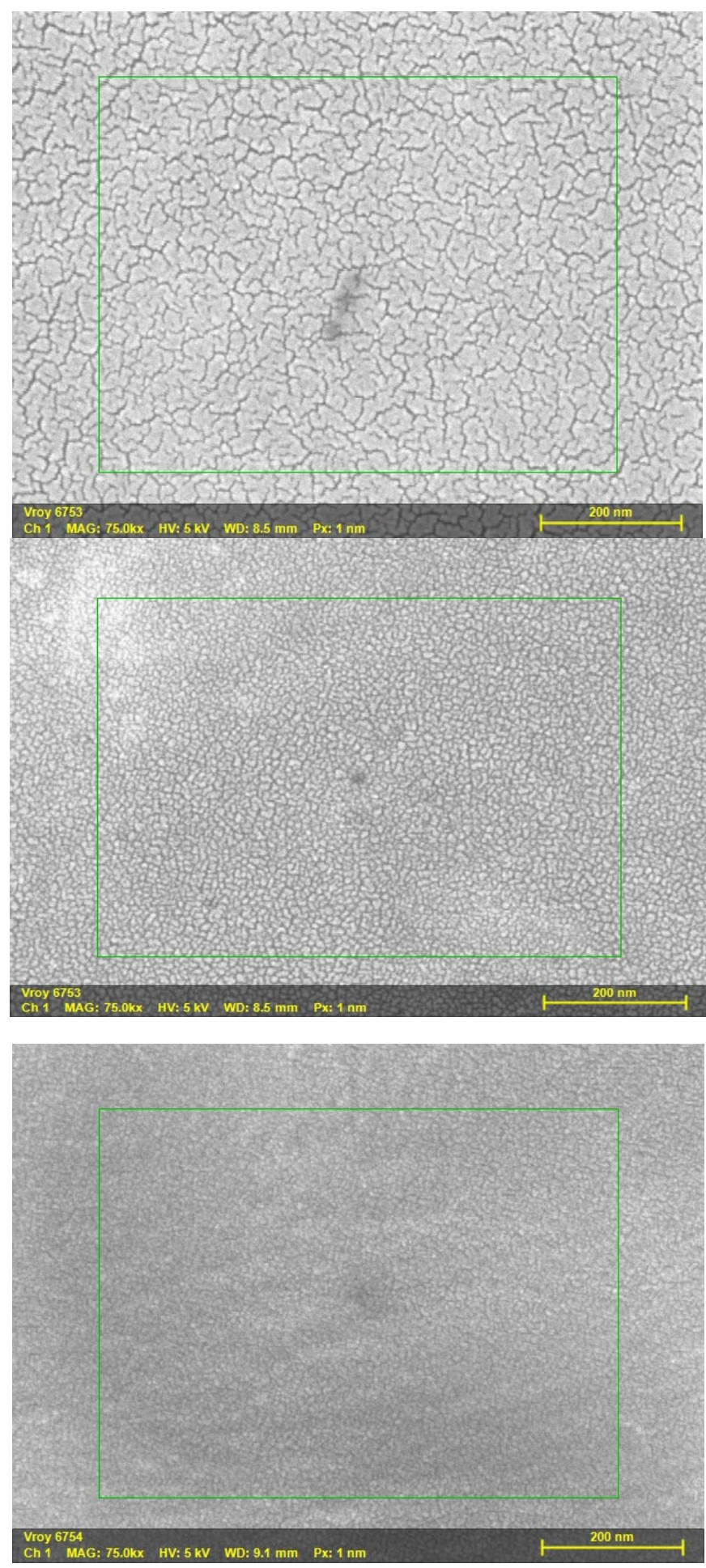

Figure S. 9. Scanning electron microscope images of surface of control ABL (top), ABL+2pr $\mathrm{TphCl}$ (middle), and $\mathrm{ABL}+2 \mathrm{pr} \mathrm{TmCl}$ (bottom), indicating progressively finer surface texture. 

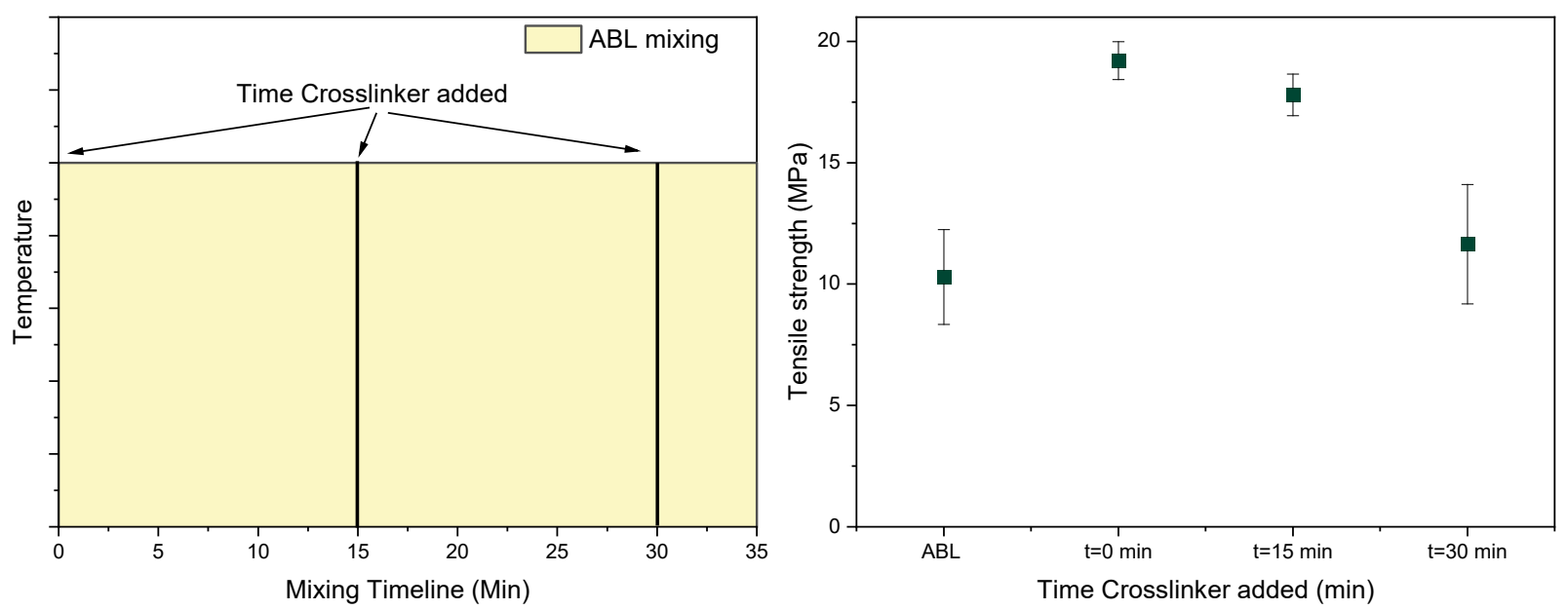

Figure $\mathrm{S}$. 10. Tensile properties of $\mathrm{ABL}+10 \mathrm{pr} \mathrm{SbCl}$ vs time at which crosslinker is added to the system, at $\mathrm{t}=0,15$ and 30 minutes in a 35-minute mixing cycle

(1) Kremer, K.; Grest, G. S. Dynamics of Entangled Linear Polymer Melts: A MolecularDynamics Simulation. J. Chem. Phys. 1990, 92 (8), 5057-5086. https://doi.org/10.1063/1.458541.

(2) Martinez, L.; Andrade, R.; Birgin, E. G.; Martínez, J. M. PACKMOL: A Package for Building Initial Configurations for Molecular Dynamics Simulations. J. Comput. Chem. 2009, 30 (13), 2157-2164. https://doi.org/10.1002/jcc.21224. 